\title{
The HKIE Best Transactions Paper Prize 2021
}

The HKIE Best Transactions Paper Prize 2021 is awarded to distinguished papers nominated from the papers published in Volume 27 Number 2, Volume 27 Number 3 and Volume 28 Number 1 of HKIE Transactions. The prize is composed of a plaque, a certificate and a cash prize of $\mathrm{HK} \$ 5,000$ for the awarded paper.

This year, the HKIE Best Transactions Paper Prize are awarded to:

"An optimised multiple kernel learning support vector machine (SVM) classification based on the fireworks algorithm and its application to the fault diagnosis of gearbox"

by Bin Yong, Jin Chen, Zuqiang Su, Xiaoping Pang and Xinghua Yang

(published in HKIE Transactions Volume 27 Number 2)

The paper can be accessed at: https://www.hkie.org.hk/hkietransactions/article_detail.php?id=846

"Chillers of air-conditioning systems: An overview"

by Michael Kwok Hi Leung, Chi Yan Tso, Wei Wu, Zhanying Zheng and Jingyu Cao

(published in HKIE Transactions Volume 27 Number 3)

The paper can be accessed at: https://www.hkie.org.hk/hkietransactions/article_detail.php?id=848

Congratulations to all our distinguished authors!

Readers are welcome to visit the HKIE Transactions for other papers of your interest at: http://www.hkie.org.hk/hkietransactions

Authors are invited to submit your manuscripts to the HKIE Transactions ScholarOne Manuscripts site at http://mc.manuscriptcentral.com/thie.

All papers published in HKIE Transactions will automatically enter the annual selection process of the HKIE Best Transactions Paper Prize. Up to three prizes will be given annually, and only if there are outstanding papers which warrant the awards. The Panel of Judges reserves the right to withhold any or all of the awards at their absolute discretion. 